\title{
FINITE-VOLUME SOLVERS FOR A MULTILAYER SAINT-VENANT SYSTEM
}

\author{
EMmanuel AUdUSSE*, MARIE-OdILE BRISTEAU ** \\ * Université Paris 13, 99 avenue J.B. Clément, 93430 Villetaneuse, France \\ e-mail: audusse@math.univ-paris13.fr \\ ** INRIA, Project Bang, Domaine de Voluceau, 78153 Le Chesnay, France \\ e-mail: marie-odile.bristeau@inria.fr
}

\begin{abstract}
We consider the numerical investigation of two hyperbolic shallow water models. We focus on the treatment of the hyperbolic part. We first recall some efficient finite volume solvers for the classical Saint-Venant system. Then we study their extensions to a new multilayer Saint-Venant system. Finally, we use a kinetic solver to perform some numerical tests which prove that the 2D multilayer Saint-Venant system is a relevant alternative to 3D hydrostatic Navier-Stokes equations.
\end{abstract}

Keywords: Saint-Venant system, shallow water equations, finite volumes, kinetic solver, approximate Riemann solvers, multilayer model

\section{Introduction}

In this article we are interested in the numerical study of shallow water flows. We mainly consider two SaintVenant type systems: the classical Saint-Venant system introduced in (de Saint-Venant, 1971) and the multilayer Saint-Venant system introduced in (Audusse, 2005). Both systems are hyperbolic systems of first order conservation laws with source terms

$$
\partial_{t} \mathbf{U}+\nabla \cdot \mathbf{F}(\mathbf{U})=\mathbf{S}(\mathbf{x}, \mathbf{U}) .
$$

The classical Saint-Venant system is commonly used for numerical simulation of various geophysical shallowwater flows, such as rivers, lakes or coastal areas, or even oceans, atmosphere or avalanches when completed with appropriate terms. Topographic, friction, viscous or Coriolis source terms may be included in the model depending on applications. The classical Saint-Venant system can be introduced using physical arguments (de Saint-Venant, 1971), but it can also be derived as a formal first-order approximation of the three-dimensional free surface incompressible Navier-Stokes equations using the so-called shallow water assumption (Ferrari and Saleri, 2004; Gerbeau and Perthame, 2001).

The multilayer Saint-Venant system, which was introduced through such an asymptotic analysis of the Navier-Stokes equations (Audusse, 2005), is a set of coupled modified Saint-Venant systems. It allows us to consider nonconstant velocities along the vertical direction (that is the main restriction of the classical SaintVenant system) while keeping the computational advantages of the classical Saint-Venant system (robustness, efficiency, etc.). It was proved to be a relevant alternative to the use of hydrostatic Navier-Stokes equations (Audusse et al., 2006a; Audusse et al., 2007). Some new source terms appear in the multilayer Saint-Venant system. In particular, it includes a nonconservative pressure source term that involves some numerical difficulties (Audusse, 2005; Audusse et al., 2006a; Audusse et al., 2007).

In this article we focus on the numerical treatment of the hyperbolic part of both classical and multilayer SaintVenant systems. This means that we are mainly concerned with the left-hand side of the system (1). We choose to work in a finite volume framework (Bouchut, 2002; Godlewski and Raviart, 1996; LeVeque, 1992). This method is very well-adapted to the numerical discretization of such conservation laws. It ensures some discrete conservative properties and it allows us to deal with discontinuous solutions that may appear in the continuous problem.

Many finite volume solvers have been developed in the last decades for the classical Saint-Venant system. Here we consider three of them, namely, the Roe, HLLE and kinetic solvers. The Roe solver (Roe, 1981; Bermudez and Vazquez, 1994) is probably most commonly used. 
The HLLE (Einfeldt et al., 1991; George, 2004) and kinetic (Audusse and Bristeau, 2005; Perthame, 2002; Perthame and Simeoni, 2001) solvers are interesting because they ensure important discrete stability properties. Our main objective in this work is to study their extension to the multilayer Saint-Venant system. The analogy between both Saint-Venant type systems makes this question quite natural. Nevertheless, some minor differences in the hyperbolic part make the answer not that clear. In the following, we show that the kinetic solver has a very natural multilayer extension while this question is quite challenging for the Roe and HLLE solvers.

Let us specify that the presence of the source term $\mathbf{S}(\mathbf{x}, \mathbf{U})$ on the right-hand side of the system (1) may also lead to numerical difficulties. These questions are not adressed here. A long list of publications is devoted to the treatment of the topographic source term. We refer to (Audusse and Bristeau, 2005) and references therein for more details. More recently some attention has also been paid to the treatment of the Coriolis source term (Audusse et al., 2006b; Bouchut et al., 2004). We also mention that the pressure source term of the multilayer SaintVenant system has to be discretized carefully (Audusse $e t$ al., 2006a; Audusse et al., 2007).

The outline of the paper is as follows: In Section 2 we introduce both classical and multilayer Saint-Venant systems. In Section 3 we recall the kinetic interpretation of the classical Saint-Venant system, and we briefly present the Roe, HLLE and kinetic solvers. In Section 4 we proceed with the multilayer Saint-Venant system, and we discuss extensions of Roe, HLLE and kinetic solvers. In Section 5 we finally present some numerical results that highlight the capabilities of the Saint-Venant multilayer system when an efficient finite volume solver is considered.

\section{Saint-Venant Type Systems}

2.1. Equations. We first introduce the classical 2D single-layer Saint-Venant system, written here in its physical conservative form

$$
\begin{aligned}
\frac{\partial h}{\partial t}+\nabla \cdot(h \mathbf{u}) & =0, \\
\frac{\partial h \mathbf{u}}{\partial t}+\nabla \cdot(h \mathbf{u} \otimes \mathbf{u})+\nabla\left(\frac{1}{2} g h^{2}\right)+g h \nabla Z & =0,
\end{aligned}
$$

where $h(t, x, y) \geq 0$ is the water height, $\mathbf{u}(t, x, y)=$ $(u, v)^{T}$ means the flow velocity, $g$ stands for the acceleration due to the gravity intensity and $Z(x, y)$ signifies the bottom depth, and therefore $h+Z$ is the water surface level (see Fig. 1). We also write $\mathbf{q}(t, x, y)=\left(q_{x}, q_{y}\right)^{T}=$ $h(t, x, y) \mathbf{u}(t, x, y)$ for the flux of water.

We also consider the 2D multilayer Saint-Venant system which is written as a set of modified single-layer

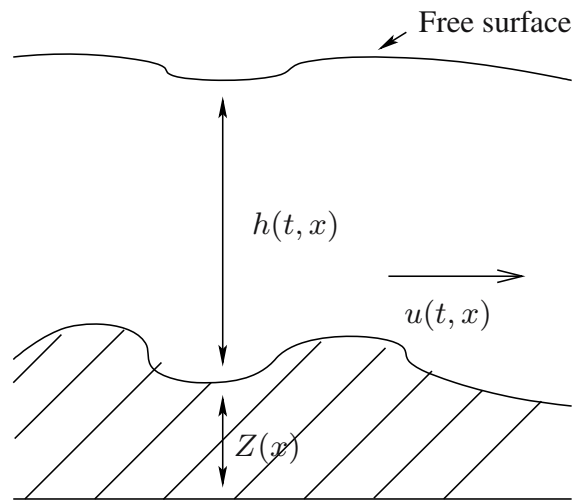

Fig. 1. Saint-Venant approach to a 1D shallow water flow.

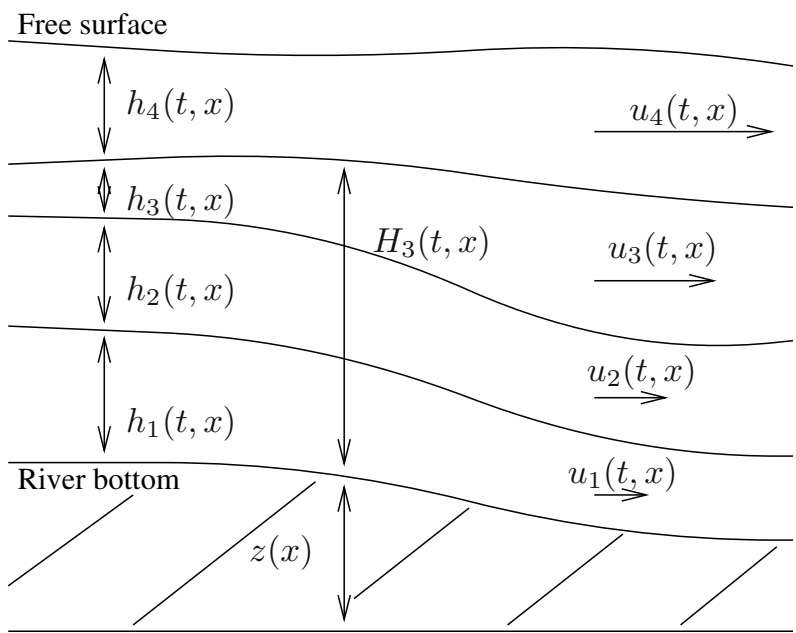

Fig. 2. Multilayer Saint-Venant approach to a $1 \mathrm{D}$ shallow water flow.

Saint-Venant systems. The number $M$ of modified singlelayer Saint-Venant systems is equal to the number of the layers that we consider in the fluid (see Fig. 2). For each $\alpha=1, \ldots, M$ we have

$$
\begin{aligned}
& \frac{\partial h_{\alpha}}{\partial t}+\nabla \cdot\left(h_{\alpha} \mathbf{U}_{\alpha}\right)=0, \\
& \frac{\partial h_{\alpha} \mathbf{U}_{\alpha}}{\partial t}+\nabla \cdot\left(h_{\alpha} \mathbf{U}_{\alpha} \otimes \mathbf{U}_{\alpha}\right)+\frac{g}{2} \nabla\left(h_{\alpha} h\right) \\
& =\frac{g}{2} h^{2} \nabla\left(\frac{h_{\alpha}}{h}\right)-g h_{\alpha} \nabla Z-\kappa_{\alpha} \mathbf{U}_{\alpha} \\
& \quad+2 \mu_{\alpha} \frac{\mathbf{U}_{\alpha+1}-\mathbf{U}_{\alpha}}{h_{\alpha+1}+h_{\alpha}}-2 \mu_{\alpha-1} \frac{\mathbf{U}_{\alpha}-\mathbf{U}_{\alpha-1}}{h_{\alpha}+h_{\alpha-1}},
\end{aligned}
$$

with

$\kappa_{\alpha}=\left\{\begin{array}{l}\kappa \text { if } \alpha=1, \\ 0 \text { if } \alpha \neq 1,\end{array} \quad \mu_{\alpha}=\left\{\begin{array}{l}0 \text { if } \alpha=0, \\ \mu \text { if } \alpha=1, \ldots, M-1, \\ 0 \text { if } \alpha=M,\end{array}\right.\right.$

where $h_{\alpha}(t, x, y) \geq 0$ is the water height of the layer $\alpha$ $\mathbf{u}_{\alpha}(t, x, y)=\left(u_{\alpha}, v_{\alpha}\right)^{T}$ is the velocity of the layer $\alpha$, and 
$\kappa$ and $\mu$ denote the bottom friction and viscosity coefficients, respectively. Thus

$$
h(t, x, y)=\sum_{\alpha=1}^{M} h_{\alpha}(t, x, y)
$$

denotes the total water height of the flow. Note that when $\alpha=1$, the multilayer system reduces to the classical Saint-Venant system.

Both systems can be derived from free surface incompressible Navier-Stokes equations under the classical shallow water assumption when a first-order approximation is considered and a (layer by layer for the multilayer system) vertical integration is applied. The shallow water assumption means here that the ratio of the characteristic height of the flow by a characteristic wave length is a small parameter $\epsilon$. The viscosity $\mu$, the friction coefficient $\kappa$ and the derivative of the bottom topography $Z(x)$ are of the order of $\epsilon$. We refer to (Ferrari and Saleri, 2004; Gerbeau and Perthame, 2001) for the derivation of the classical Saint-Venant system and to (Audusse, 2005; Audusse et al., 2006a; Audusse et al., 2007) for the derivation of the multilayer Saint-Venant system. In the next subsection we shall recall important properties of both systems and explain the choice of the particular form of the multilayer Saint-Venant system that we consider.

2.2. Properties of Saint-Venant Type Systems. The systems (2), (3) and (4), (5) can be written in the general form

$$
\frac{\partial \mathbf{U}}{\partial t}+\nabla \cdot \mathbf{F}(\mathbf{U})=\mathbf{S}(x, \mathbf{U}),
$$

where $\mathbf{U}$ is the vector of conservative variables, $\mathbf{F}(\mathbf{U})$ constitutes the conservative flux term and $\mathbf{S}(x, \mathbf{U})$ the nonconservative source term.

Both systems (2), (3) and (4), (5) admit an invariant region $h(t, x) \geq 0$. The preservation of this property at the discrete level is fundamental and is not achieved with all finite volume solvers.

Both systems (2), (3) and (4), (5) are hyperbolic for $h>0$. This means that the flux Jacobian is diagonalizable with real eigenvalues. They are strictly hyperbolic when single- or two-layer Saint-Venant systems are considered (see (Audusse, 2005) for more details). The particular choice of the multilayer system (4), (5) is partially related to this property. In (Castro et al., 2001), a slightly different two-fluid system is considered that turns to be nonhyperbolic in its two-layer form (i.e., when fluids with the same densities are considered). The water height $h$ can vanish (flooding zones, dry regions, tidal flats) and the systems lose hyperbolicity at $h=0$, which implies theoretical and numerical difficulties. Finally, both systems are also concerned with a fundamental entropy inequality for the physical energy (Audusse, 2005; Audusse and Bristeau, 2005; George, 2004; Perthame and Simeoni, 2001).

\section{Finite-Volume Solvers for the Classical Saint-Venant System}

Here we are interested in the numerical discretization of the hyperbolic part of the system (6). A possible numerical treatment of the source term $\mathbf{S}(\mathbf{x}, \mathbf{U})$ is briefly described in Section 5. The difficulty in defining accurate numerical schemes for the systems (2), (3) or (4), (5) is related to the deep mathematical structure of such hyperbolic systems. For the classical Saint-Venant system (2), (3), the first existence proof of weak solutions after shocks in the large is due to (Lions et al., 1996). It is based on the kinetic interpretation of the system, which is also a method to derive numerical schemes with good properties.

It is important to get schemes that satisfy very natural properties such as the conservation and nonnegativity of the water height, the ability to compute dry areas, and eventually satisfy a discrete entropy inequality. In the last decade many works have been devoted to this question and a large choice of schemes is now available for the system (2), (3). Few of them satisfy all of the required stability properties. This is the case for the HLLE scheme (Einfeldt et al., 1991; George, 2004) or for kinetic schemes (Audusse and Bristeau, 2005; Perthame and Simeoni, 2001). The Roe scheme (Roe, 1981; Bermudez and Vazquez, 1994) is one of the most commonly used schemes but does not ensure the positivity of the discrete water height, cf. (Benkhaldoun et al., 1999) for more details on this question. Let us now give a brief overview of the Roe, HLLE and kinetic schemes applied the classical Saint-Venant system before we consider their extension to the multilayer system (4), (5) in the next subsection.

3.1. Approximate Riemann Solvers. The first attempt to propose a finite-volume solver for hyperbolic systems of type (6) is due to Godunov (1959). Starting from a piecewise constant solution, he proposed to solve the exact Riemann problem at each interface and then to consider the mean value of the solution at the end of the time step in order to construct a piecewise initial solution for the next time step. This scheme is precise but computationally expensive. Some authors introduced what is called the approximate Riemann solver. It consists in computing a solution to an approximate Riemann problem instead of the exact one. Both Roe and HLLE solvers are approximate Riemann solvers.

Note that a classical way to treat $2 \mathrm{D}$ problems in a finite-volume framework is to successively consider a planar 1D problem in the normal direction to each cell interface. Considering a cell $C_{i}$ we make a loop on the cell interfaces $\Gamma_{i j}$, where $j$ denotes the neighbouring cells $C_{j}$. Considering the interface $\Gamma_{i j}$ we switch from the reference frame $\left(e_{x}, e_{y}\right)$ to the local frame that is defined by $\left(e_{n_{i j}}, e_{\tau_{i j}}\right)$, where the subscripts $n_{i j}$ and $t_{i j}$ denote the normal and tangential directions to $\Gamma_{i j}$, respec- 
tively. We solve a 1D problem in this local frame and we come back to the reference frame $\left(e_{x}, e_{y}\right)$ in order to add the contributions of all cell interfaces $\Gamma_{i j}$. For more details about this computational method, we refer the reader to (Audusse and Bristeau, 2005; Bristeau and Coussin, 2001). In the following, we describe the 1D approximate Riemann solver that is used at each cell interface. All quantities are thus constant in the $\tau$-direction. We denote by $u$ the normal velocity and by $v$ the tangential velocity.

The Roe solver aims at solving the following linearized Riemann problem:

$$
\partial_{t} \mathbf{U}+A(\hat{\mathbf{U}}) \partial_{n} \mathbf{U}=0, \quad\left\{\begin{array}{l}
\mathbf{U}(0, x<0)=\mathbf{U}_{l}, \\
\mathbf{U}(0, x \geq 0)=\mathbf{U}_{r} .
\end{array}\right.
$$

This Riemann problem involves the so-called Roe matrix $A(\hat{\mathbf{U}})$ (Roe, 1981). In the case of the classical SaintVenant system, it is nothing but the flux Jacobian $D F(\mathbf{U})$ computed at some particular Roe average states $\hat{\mathbf{U}}$ which satisfy some consistency relations, cf. (14) and (15) below. The Roe average states for the classical Saint-Venant system are well known (Bristeau and Coussin, 2001):

$$
\begin{gathered}
\hat{h}=\frac{h_{l}+h_{r}}{2}, \quad \hat{u}=\frac{\sqrt{h_{l}} u_{l}+\sqrt{h_{r}} u_{r}}{\sqrt{h_{l}}+\sqrt{h_{r}}}, \\
\hat{v}=\frac{\sqrt{h_{l}} v_{l}+\sqrt{h_{r}} v_{r}}{\sqrt{h_{l}}+\sqrt{h_{r}}} .
\end{gathered}
$$

To apply the Roe solver is now nothing but to compute the solution of the linearized Riemann problem (7), (8). This requires the computation of the eigenvalues and eigenvectors of the Roe matrix. Here they are the eigenvalues and eigenvectors of the flux Jacobian. These eigenvalues are well known for the classical Saint-Venant system (Bristeau and Coussin, 2001):

$$
\lambda_{1}=u-\sqrt{g h}, \quad \lambda_{2}=u+\sqrt{g h}, \quad \lambda_{0}=u .
$$

The HLL type solvers (Harten et al., 1983) are another kind of approximate Riemann solvers. They assume a simpler wave structure than the exact Riemann solution. For application to the classical Saint-Venant system, the key point is to estimate the speed of the acoustic wave. The choice

$$
\begin{aligned}
& S_{l}=\min \left(\lambda_{1}\left(\mathbf{U}_{l}\right), \lambda_{1}(\hat{\mathbf{U}})\right), \\
& S_{r}=\max \left(\lambda_{2}\left(\mathbf{U}_{r}\right), \lambda_{2}(\hat{\mathbf{U}})\right)
\end{aligned}
$$

leads to the HLLE solver (Einfeldt et al., 1991; George, 2004). It possesses interesting stability properties such as the positivity of the water height and a discrete in-cell entropy inequality. As can be seen in (10), it needs the computation of the eigenvalues (9) of the flux Jacobian and of the Roe average states (8).
3.2. Kinetic Schemes. Kinetic schemes are based on the kinetic interpretation of shallow water models. The kinetic interpretation was first introduced for Euler equations (Khobalatte, 1993; Perthame, 2002). It consists in introducing a new variable $\xi$ that represents the velocity of the particles and a new function $M$ that denotes the density of particles in the phase space. Let us first detail its form when the 2D classical Saint-Venant system (2), (3) is considered:

$$
\begin{aligned}
M(t, x, y, \xi) & =M(h, \xi-\mathbf{u}) \\
& =\frac{h(t, x, y)}{\tilde{c}^{2}} \chi\left(\frac{\xi-\mathbf{u}(t, x, y)}{\tilde{c}}\right),
\end{aligned}
$$

where $\chi$ is an even compactly supported probability function with appropriate properties (Audusse and Bristeau, 2005), and

$$
\tilde{c}=\sqrt{\frac{1}{2} g h} .
$$

The 2D macroscopic Saint-Venant system can be obtained after the integration of a Boltzmann type equation for the density function $M$ :

$$
\frac{\partial M}{\partial t}+\xi \cdot \nabla_{\mathbf{x}} M=Q(t, x, y, \xi),
$$

where $Q(t, x, y, \xi)$ is a collision term for which the first two moments are equal to zero. It can be proved (Audusse and Bristeau, 2005) that $(h, h \mathbf{u})$ is a solution of the classical Saint-Venant system (2), (3) if and only if $M$ is a solution of the kinetic equation (12).

This property produces a very useful numerical consequence: the nonlinear system (2), (3) can be viewed as a linear transport equation on a nonlinear quantity $M$, for which it is easy to find a simple numerical scheme with good theoretical properties. In particular, it is easy to ensure the positivity of the discrete density function $M$. Then a simple integration of this microscopic scheme allows us to derive a macroscopic scheme for the classical Saint-Venant system (2), (3). The positivity of the discrete water height is thus obviously preserved since the water height is the integral of the density function $M$.

The $\chi$ function (11) that is introduced in the kinetic interpretation is not unique. Different choices of this function lead to different kinetic solvers. In particular, a discrete entropy inequality can be proved for a particular choice of the $\chi$ function (Perthame and Simeoni, 2001). For a precise description of 2D kinetic schemes for the classical Saint-Venant system, we refer the reader to (Audusse and Bristeau, 2005) where a detailed implementation is also presented.

\section{Finite-Volume Solvers for the Multilayer Saint-Venant System}

Now let us consider the multilayer Saint-Venant system (4), (5). Here we are concerned only with the hyperbolic 
left-hand side. It is composed of a system of $2 M$ coupled equations with $2 M$ unknowns: one water height and one velocity for each one of the $M$ layers. The coupling on the left-hand side is only due to the total water height that appears in the conservative pressure term of the momentum equation (5). It follows that for any layer the advective flux term is the same as the classical one, i.e.,

$$
\nabla \cdot\left(h_{\alpha} \mathbf{u}_{\alpha} \otimes \mathbf{u}_{\alpha}\right)
$$

This is not the case for the pressure flux term. Instead of involving only one water height $h$ as for the classical Saint-Venant system,

$$
\nabla\left(\frac{1}{2} g h^{2}\right)
$$

it now involves $M$ different water heights: the water height of the layer we consider (as in the classical SainVenant system), but also all the water heights of the $M-1$ other layers:

$$
\nabla\left(\frac{1}{2} g h_{\alpha} \sum_{\beta=1}^{M} h_{\beta}\right) .
$$

Hence we cannot directly extend the methods that have been presented in the previous subsection for the classical Saint-Venant system to this new problem. For numerical purposes it would be easier to consider a multilayer model where, for each layer, the flux term would be identical to the flux term of the classical Saint-Venant system

$$
\nabla\left(\frac{1}{2} g h_{\alpha}^{2}\right)
$$

In this case the whole coupling effect due to the pressure term is on the right-hand side. Such a system can also be obtained by the same procedure and the left-hand side can be proved to be hyperbolic. Moreover, it would allow us to use the classical Roe, HLLE or kinetic schemes for each layer separately. Nevertheless, it would lead to nonphysical solutions as observed in (Audusse, 2005; Castro et al., 2001). The main reason is that the eigenvalues of the flux Jacobian of the hyperbolic part of such a system do not contain the right physical information. In particular, the barotropic eigenvalues (16) are not recovered.

The problem is now to construct a numerical scheme for the multilayer Saint-Venant system (4), (5). In particular, we are interested in knowing if we can use a simple extension of classical schemes designed for the classical Saint-Venant system. In this subsection we discuss this question for the Roe, HLLE and kinetic schemes.

4.1. Approximate Riemann Solvers for the Multilayer System. Let us begin with the study of the Roe and HLLE schemes. The first step here is the definition of Roe average states for the multilayer case.
Proposition 1. The Roe average states of the multilayer system (4), (5) are a simple extension of the Roe average states (8) of the classical Saint-Venant system

$$
\begin{gathered}
\hat{h}_{\alpha}=\frac{h_{\alpha l}+h_{\alpha r}}{2}, \quad \hat{u}_{\alpha}=\frac{\sqrt{h_{\alpha l}} u_{\alpha l}+\sqrt{h_{\alpha r}} u_{\alpha r}}{\sqrt{h_{\alpha l}}+\sqrt{h_{\alpha r}}}, \\
\hat{v}_{\alpha}=\frac{\sqrt{h_{\alpha l}} v_{\alpha l}+\sqrt{h_{\alpha r}} v_{\alpha r}}{\sqrt{h_{\alpha l}}+\sqrt{h_{\alpha r}}} .
\end{gathered}
$$

Proof. Since the Roe matrix $A$ is here the exact Jacobian of the physical flux $F$, it is sufficient to ensure that the Roe average states (13) satisfy for each $\alpha=1, \ldots, M$

$$
\begin{gathered}
\hat{h}_{\alpha}\left(\mathbf{U}_{\alpha}, \mathbf{U}_{\alpha}\right)=h_{\alpha}, \quad \hat{u}_{\alpha}\left(\mathbf{U}_{\alpha}, \mathbf{U}_{\alpha}\right)=u_{\alpha}, \\
\hat{v}_{\alpha}\left(\mathbf{U}_{\alpha}, \mathbf{U}_{\alpha}\right)=v_{\alpha}
\end{gathered}
$$

and that the Roe matrix $D F(\hat{\mathbf{U}})$ computed from the Roe states (13) satisfies the consistency relation

$$
\mathbf{F}\left(\mathbf{U}_{r}\right)-\mathbf{F}\left(\mathbf{U}_{l}\right)=D F(\hat{\mathbf{U}})\left(\mathbf{U}_{r}-\mathbf{U}_{l}\right) .
$$

The first relation (14) is obvious. The proof for the second one (15) just needs an easy computation.

For both Roe and HLLE schemes it is also needed to compute the eigenvalues of the Roe matrix. Here they are nothing but the eigenvalues of the flux Jacobian. In (Audusse, 2005), a first-order approximation of these eigenvalues is given for the two-layer case under the hypothesis that the two-layer velocities are close to mean velocity $u$

$$
\begin{aligned}
& \lambda_{\text {ext }}^{ \pm}=u_{m} \pm \sqrt{g\left(h_{1}+h_{2}\right)}+O\left(\left|u_{1}-u\right|^{2},\left|u_{2}-u\right|^{2}\right), \\
& \lambda_{\text {int }}^{ \pm}=u_{c} \pm \sqrt{\frac{g\left(h_{1}+h_{2}\right)}{2}}+O\left(\left|u_{1}-u\right|^{2},\left|u_{2}-u\right|^{2}\right), \\
& \lambda_{\mathrm{tr}}^{1}=u_{1}, \quad \lambda_{\mathrm{tr}}^{2}=u_{2},
\end{aligned}
$$

where

$$
u_{m}=\frac{h_{1} u_{1}+h_{2} u_{2}}{h_{1}+h_{2}}, \quad u_{c}=\frac{h_{1} u_{2}+h_{2} u_{1}}{h_{1}+h_{2}} .
$$

The eigenvalues $\lambda_{\text {ext }}^{ \pm}$are barotropic quantities since they are related to surface waves. The eigenvalues $\lambda_{\text {int }}^{ \pm}$ are related to internal interface waves and thus are called baroclinic (Castro et al., 2001). The eigenvalues $\lambda_{\mathrm{tr}}^{1,2}$ are concerned with a simple transport process of the tangential velocity in each layer. Note that a zeroth-order approximation of the barotropic eigenvalues (16) reduces to the eigenvalues of the classical Saint-Venant system (9).

In (Castro et al., 2001), the authors consider a slightly different bifluid Saint-Venant system. They also compute an approximation of the eigenvalues of the Roe matrix under the same hypothesis. In the case of a twofluid problem this system contains more relevant information than the multilayer system that we consider here 
(Audusse, 2005). But when a single fluid is considered, its two-layer version turns to be nonhyperbolic since it leads to complex baroclinic eigenvalues when the densities of both the layers are equal (Castro et al., 2001).

Since we are interested in these eigenvalues in order to use Roe or HLLE schemes in a fully multilayer case, let us go one step further in the analysis by considering a real multilayer case with an arbitrary number of layers.

Proposition 2. Consider the multilayer Saint-Venant system (4), (5) with $M$ layers. We suppose that all the velocities $\left(u_{\alpha}\right)_{\alpha=1, \ldots, M}$ are closed to a mean velocity $u$. A first-order approximation of the two barotropic eigenvalues is given by

$$
\lambda_{\mathrm{ext}}^{ \pm}=u_{m} \pm \sqrt{g \sum_{\alpha=1}^{M} h_{\alpha}+O\left(\left|u_{\beta}-u\right|^{2}\right)_{\beta=1, \ldots, M}}
$$

where

$$
u_{m}=\frac{\sum_{\alpha} h_{\alpha} u_{\alpha}}{\sum_{\alpha} h_{\alpha}} .
$$

The 2(M-1) barotropic eigenvalues (associated with $M-1$ interfaces) have the following zeroth-order approximations:

$$
\begin{array}{r}
\lambda_{\text {int }}^{ \pm, \alpha+\frac{1}{2}}=u \pm \sqrt{\frac{1}{2} g \sum_{\alpha=1}^{M} h_{\alpha}}+O\left(\left|u_{\beta}-u\right|\right)_{\beta=1, \ldots, M} \\
\alpha=1, \ldots, M-1
\end{array}
$$

Finally, there are $M$ transport eigenvalues

$$
\lambda_{\mathrm{tr}}^{\alpha}=u_{\alpha}, \quad \alpha=1, \ldots, M
$$

Proof. Here also the proof relies on technical computations. An iterative procedure can be used by starting from the result for the two-layer system (16) and (17).

Remark 1. The first order approximation of the baroclinic eigenvalues depends on the number of the layers that are considered. In particular, when a three-layer system is considered, the formula (20) is a first-order approximation to the four baroclinic eigenvalues.

When the assumptions of Proposition 2 are fullfilled, two groups of $M-1$ barotropic eigenvalues are very close to each other. This may lead to numerical difficulties when evaluating the eigenvalues and eigenvectors of the Roe matrix. Now if the flow is such that the hypothesis on the velocities is not satisfied, we cannot apply Roe or HLLE solvers by using formulas (19) and (20). In any case note that the approximation of the barotropic eigenvalues (20) is just a zeroth-order approximation and then is not so good. Moreover, we also have to compute the corresponding eigenvectors if we want to use a Roe solver.
In (Castro et al., 2001) the authors give some ways to compute the eigenvalues more precisely. Nevertheless, it makes the extension of Roe or HLLE solvers to multilayer system not obvious. Moreover, the computational cost of the computation of the eigenvalues becomes quite large.

\subsection{Kinetic Schemes for the Multilayer Saint-Venant}

System. Let us now turn to the extension of the kinetic solver to the multilayer system (4) and (5). The key point here is to find a kinetic representation of the multilayer system. As was the case for the computation of the Roe matrix, such a representation is an obvious extension of the classical one

Proposition 3. We can represent the multilayer system (4) and (5) through the following $M$ kinetic equations:

$$
\frac{\partial M_{\alpha}}{\partial t}+\xi \cdot \nabla_{\mathbf{x}} M_{\alpha}=Q_{\alpha}(t, x, y, \xi),
$$

where the density function $M_{\alpha}$ is defined by

$$
\begin{aligned}
M_{\alpha}(t, x, y, \xi) & =M_{\alpha}\left(\left(h_{\beta}\right)_{\beta=1, \ldots, M}, \xi-\mathbf{u}_{\alpha}\right) \\
& =\frac{h_{\alpha}(t, x, y)}{\tilde{c}^{2}} \chi\left(\frac{\xi-\mathbf{u}_{\alpha}(t, x, y)}{\tilde{c}}\right)
\end{aligned}
$$

with

$$
\tilde{c}=\sqrt{\frac{1}{2} g \sum_{\beta} h_{\beta}}
$$

Proof. The integration of the kinetic equations (23) for 1 and $\xi$ gives the mass and momentum equations (4) and (5), respectively.

Observe that the kinetic representation of the multilayer Saint-Venant system is very close to the kinetic representation of the classical Saint-Venant system introduced in the previous subsection. The only difference is that the water heights that appear inside and outside the $\chi$ function are no more the same. The reason for that is that the velocity $\tilde{c}$ has to be defined from the total water height and not from the layer water height. This is related to the fact that the expression of the barotropic eigenvalues (19) involves the total water height, see (Audusse, 2005) for more details.

From this kinetic interpretation it is then very easy to derive a kinetic scheme for the multilayer Saint-Venant system. As for the classical case, it is sufficient to apply an upwind scheme to each kinetic equation and then to integrate this scheme on the velocity variable. Finally, we obtain a macroscopic scheme with the required stability properties.

\section{Numerical Applications}

Here we present two numerical tests. The first one is a 1D dam break test that highlights the fact that the multilayer 
Saint-Venant system (4) and (5) gives good results (in the sense that they are in good agreement with Navier-Stokes solutions) even for cases where the shallow water assumption is not fullfilled. Namely, we consider a no-slip condition on the bottom. It is equivalent to choosing an infinite friction coefficient $\kappa$ in (5). Let us also precise that the classical Saint-Venant system cannot be used in this case since this will lead to a zero velocity for the entire flow. When the multilayer Saint-Venant system is used, this test leads to a vertical velocity profile (see Fig. 4) for which the horizontal velocities of the different layers are not close to the mean velocity since the velocity of the lowest layer is equal to zero. The assumptions of Proposition 2 are thus not fullfilled and the approximated eigenvalues (19) and (20) are not relevant. The use of an extension of the Roe or HLLE solvers is particularly difficult in this case. Here the computation is performed with the kinetic solver introduced in Section 4.2. The non-conservative pressure source term of the momentum equation (5) is treated explicitly in a finite volume framework. We refer the reader to (Audusse et al., 2006a; Audusse et al., 2007) for more details. The viscous source term is computed implicitly. It leads to the solution of an invertible tridiagonal linear system. The Navier-Stokes computation is based on an implicit ALE method. Both computations are performed with ten layers. The free surface profile computed with the multilayer model is presented in Fig. 3. When the friction coefficient is equal to zero, the free surface is flat between the rarefaction wave (Fig. 3 on the left) and the schock wave (Fig. 3 on the right). One of the effects of the friction is that this zone presents a slight slope. In Fig. 4 we then present the vertical profiles of the horizontal velocity at some point in this zone. Here we choose $x=10$, but the profile is almost the same everywhere. the Multilayer Saint-Venant and Navier-Stokes solutions appear to be in good agreement. We also perform multilayer computations with only five layers. The comparison with the

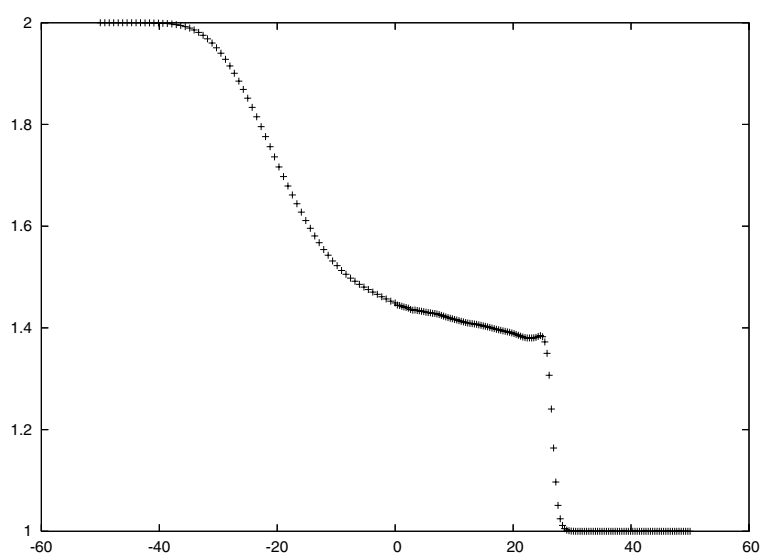

Fig. 3. Free surface (longitudinal profile): a multilayer SaintVenant model with ten layers and no slip.

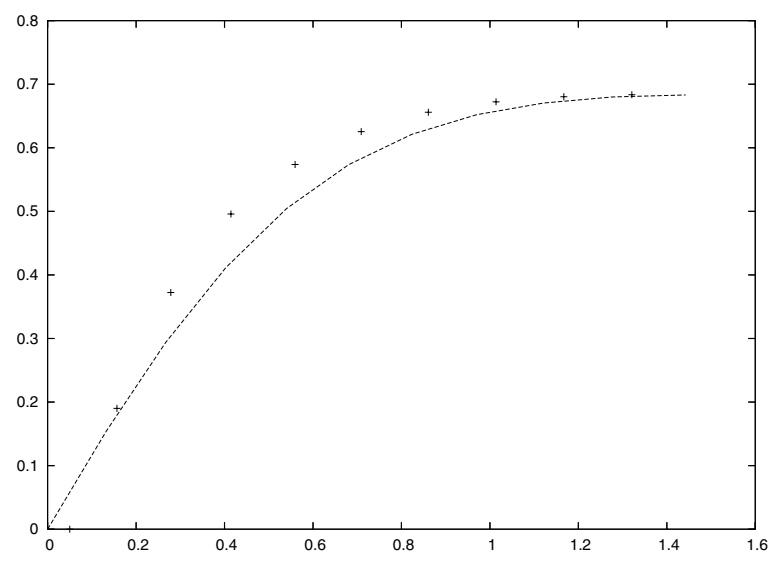

Fig. 4. Velocity (vertical profiles): the Navier-Stokes (continuous line) and multilayer Saint-Venant (crosses) models with ten layers and no slip.

Navier-Stokes solution is presented in Fig. 5 (top). Here too the two solutions are in good agreement. Even with a two-layer version of the system, the velocity of the upper layer appears to be a quite good approximation of the velocity of the upper part of the flow, see Fig. 5.

The second numerical example is a $2 \mathrm{D}$ test case of a stationary flow over a bump. It includes a transcritical transition between sub- and supersonic zones and the presence of a stationary shock wave. Here we compare the solution of the multilayer Saint-Venant system (4), (5) and of hydrostatic Navier-Stokes equations. The solutions are very similar as for computational cost, which is three times smaller with the multilayer system. The numerical solver for the multilayer system is a $2 \mathrm{D}$ extension of the one we described in the previous subsection. The hydrostatic Navier-Stokes computations were performed with the Telemac code (Hervouet, 2003). Each computation was performed with six layers. The free surface profiles are presented in Fig. 6. The horizontal velocities are presented in Fig. 7.

\section{Conclusion}

In this paper we considered three well-known finite volume solvers (Roe, HLLE) and kinetic solvers that are commonly used for the computation of the classical SaintVenant system and we studied their extension to the computation of a multilayer Saint-Venant system. All the three solvers are proved to have extensions for the multilayer problem. Nevertheless, the extension of the kinetic solver is proved to be more natural and less time consuming. We also presented some numerical results that highlight the possibility of the multilayer Saint-Venant system when a kinetic solver is used. 

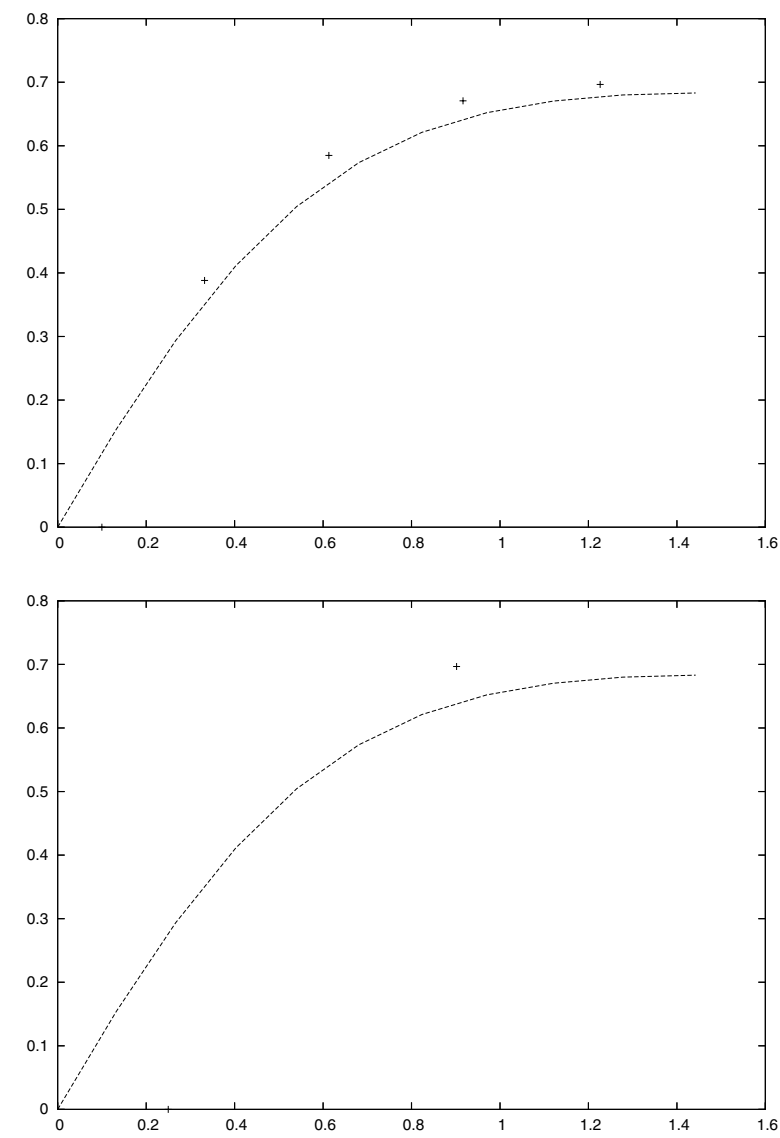

Fig. 5. Velocity (vertical profiles): the Navier-Stokes (continuous line) and multilayer Saint-Venant (crosses) models with five (top) and two (bottom) layers, no slip.

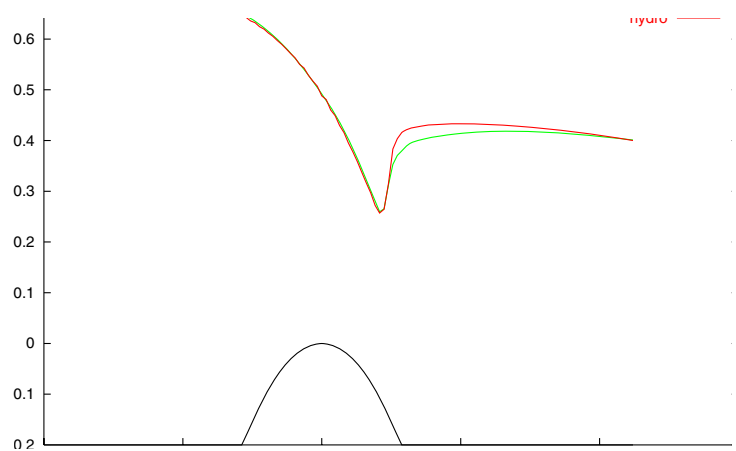

Fig. 6. Free surface comparisons. A multilayer Saint-Venant model (solid line) and a hydrostatic Navier-Stokes model (dotted line).

\section{Acknowledgments}

The authors thank J.M. Hervouet and A. Decoene for providing the results of TELEMAC computations. The authors thank J.F. Gerbeau for providing the result of 2D Navier-Stokes computations.

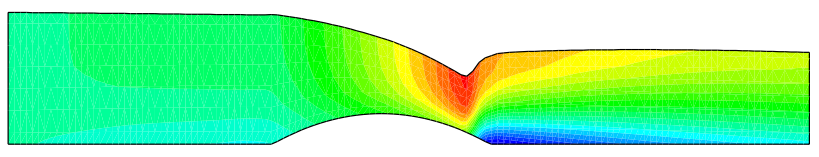

(a)

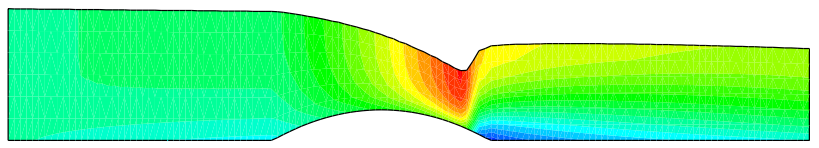

(b)

Fig. 7. Horizontal velocities: (a) a multilayer Saint-Venant model, (b) a hydrostatic Navier-Stokes model.

\section{References}

Audusse E. (2005): A multilayer Saint-Venant model. Discrete and Continuous Dynamical Systems, Series B, Vol. 5, No. 2, pp. 189-214

Audusse E. and Bristeau M.O. (2005): A well-balanced positivity preserving second order scheme for shallow water flows on unstructured meshes. Journal of Computational Physics, Vol. 206, pp. 311-333.

Audusse E., Bristeau M.O. and Decoene A. (2006a): 3D free surface flows simulations using a multilayer SaintVenant model-Comparisons with Navier-Stokes solutions. Proceedings of the 6-th European Conference Numerical Mathematics and Advanced Applications, ENUMATH 2005, Santiago de Compostella, Spain, pp. 181-189.

Audusse E., Klein R. and Owinoh A. (2006b): Conservative and well-balanced discretizations for shallow water flows on rotational domains. Proceedings of the 77th Annual Scientific Conference Gesellschaft für Angewandte Mathematik und Mechanik, GAMM 2006, Berlin, Germany.

Audusse E., Bristeau M.O. and Decoene A. (2007): Numerical simulations of $3 D$ free surface flows by a multilayer SaintVenant model. (submitted).

Bermudez A. and Vazquez M.E. (1994): Upwind methods for hyperbolic conservation laws with source terms. Computers and Fluids, Vol. 23, No. 8, pp. 1049-1071.

Benkhaldoun F., Elmahi I. and Monthe L.A. (1999): Positivity preserving finite volume Roe schemes for transportdiffusion equations. Computer Methods in Applied Mechanics and Engineering, Vol. 178, pp. 215-232.

Bouchut F. (2002): An introduction to finite volume methods for hyperbolic systems of conservation laws with source. Ecole CEA - EDF - INRIA Ecoulements peu profonds à surface libre, Octobre 2002, INRIA Rocquencourt, http://www.dma.ens.fr/ fbouchut/ publications/fvcours.ps.gz

Bouchut F., Le Sommer J. and Zeitlin V. (2004): Frontal geostrophic adjustment and nonlinear wave phenomena in one dimensional rotating shallow water. Part 2: Highresolution numerical simulations. Journal of Fluid Mechanics, Vol. 513, pp. 35-63. 
Bristeau M.O. and Coussin B. (2001): Boundary Conditions for the Shallow Water Equations solved by Kinetic Schemes. INRIA Report, Vol. 4282,

http: //www.inria.fr/RRRT/RR-4282.html

Castro M., Macias J. and Pares C. (2001): A Q-scheme for a class of systems of coupled conservation laws with source term. Application to a two-layer 1-D shallow water system. ESAIM: Mathematical Modelling and Numerical Analysis, Vol. 35, pp. 107-127.

Einfeldt B., Munz C.D., Roe P.L. and Slogreen B. (1991): On Godunov type methods for near low densities. Journal of Computational Physics, Vol. 92, pp. 273-295.

Ferrari S. and Saleri F. (2004): A new two-dimensional Shallow Water model including pressure effects and slow varying bottom topography. ESAIM: Mathematical Modelling and Numerical Analysis, Vol. 38, No. 2, pp. 211-234.

George D. (2004): Numerical Approximation of the Nonlinear Shallow Water Equations with Topography and Dry Beds: A Godunov-Type Scheme. M.Sc. Thesis, University of Washington.

Gerbeau J.-F. and Perthame B. (2001): Derivation of Viscous Saint-Venant System for Laminar Shallow Water; Numerical Validation. Discrete and Continuous Dynamical Systems, Ser. B, Vol. 1, No. 1, pp. 89-102.

Godlewski E. and Raviart P.-A. (1996): Numerical Approximation of Hyperbolic Systems of Conservation Laws. New York: Springer-Verlag.

Godunov S.K. (1959): A difference method for numerical calculation of discontinuous equations of hydrodynamics. Matematicheski Sbornik, pp. 271-300, (in Russian).
Harten A., Lax P.D. and Van Leer B. (1983): On upstream differencing and Godunov-type schemes for hyperbolic conservation laws. SIAM Review, Vol. 25, pp. 35-61.

Hervouet J.M. (2003): Hydrodynamique des écoulements à surface libre; Modélisation numérique avec la méthode des éléments finis. Paris: Presses des Ponts et Chaussées, (in French).

Khobalatte B. (1993): Résolution numérique des équations de la mécanique des fluides par des méthides cinétiques. Ph.D. Thesis, Université P. \& M. Curie (Paris 6), (in French).

LeVeque R.J. (1992): Numerical Methods for Conservation Laws. Basel: Birkhäuser.

Lions P.L., Perthame B. and Souganidis P.E. (1996): Existence of entropy solutions for the hyperbolic systems of isentropic gas dynamics in Eulerian and Lagrangian coordinates. Communications on Pure and Applied Mathematics, Vol. 49, No. 6, pp. 599-638.

Perthame B. (2002): Kinetic Formulations of Conservation Laws. Oxford: Oxford University Press.

Perthame B. and Simeoni C. (2001): A kinetic scheme for the Saint-Venant system with a source term. Calcolo, Vol. 38, No. 4, pp. 201-231.

Roe P.L. (1981): Approximate Riemann solvers, parameter vectors and difference schemes. Journal of Computational Physics, Vol. 43, pp. 357-372.

de Saint-Venant A.J.C. (1971): Théorie du mouvement nonpermanent des eaux, avec application aux crues des rivières et à l'introduction des marées dans leur lit (in French). Comptes Rendus de l'Académie des Sciences, Paris, Vol. 73, pp. 147-154. 
